\section{Radial access for percutaneous coronary procedure: relationship between operator expertise and complications}

\author{
Simona Susanu*, Marco Angelillis*, Cristina Giannini*, Rossella Binella, \\ Anna Matteoni, Rita Bellucci, Sandro Balestri, Nicola Ferrara, \\ Federico Falchi, Giuliano Micheletti, Anna Sonia Petronio
}

Catheterization Laboratory, University of Pisa, Pisa, Italy

Objective The aim of this study was to investigate (1) whether the learning curve of new catheterization laboratory operators increases the incidence of complications of transradial access during percutaneous coronary interventions and (2) whether manual compression with a twostep approach is safe and efficient for radial access hemostasis.

Methods We performed a prospective study with all consecutive patients who underwent a coronary diagnostic or intervention procedure with radial access. The primary end point was a composite of pulseless radial artery of the wrist and hematoma evaluated after 24 hours. The secondary end point of efficacy was defined as the presence of bleeding or hematoma after 30 seconds.

Results From March 2016 to June 2016, 150 consecutive patients, of whom 147 underwent coronary angiography and/or percutaneous coronary intervention through radial access, were included in the present study. The primary end point was present in 33\%, but pulseless radial artery of the wrist was present only in 5.3\%. We found that the incidence of primary end point was statistically different according to the number of puncture attempts, with a cutoff of two punctures with blood. The secondary end point of safety was present only in $4.7 \%$ of the cases.

Conclusion Radial access is feasible and safe even if performed by training physicians. Manual compression with early evaluation after 30 seconds is a safe technique for managing the radial access after sheath removal.

Keywords Vascular access; Manual compression; Attending physician; Cardiology training

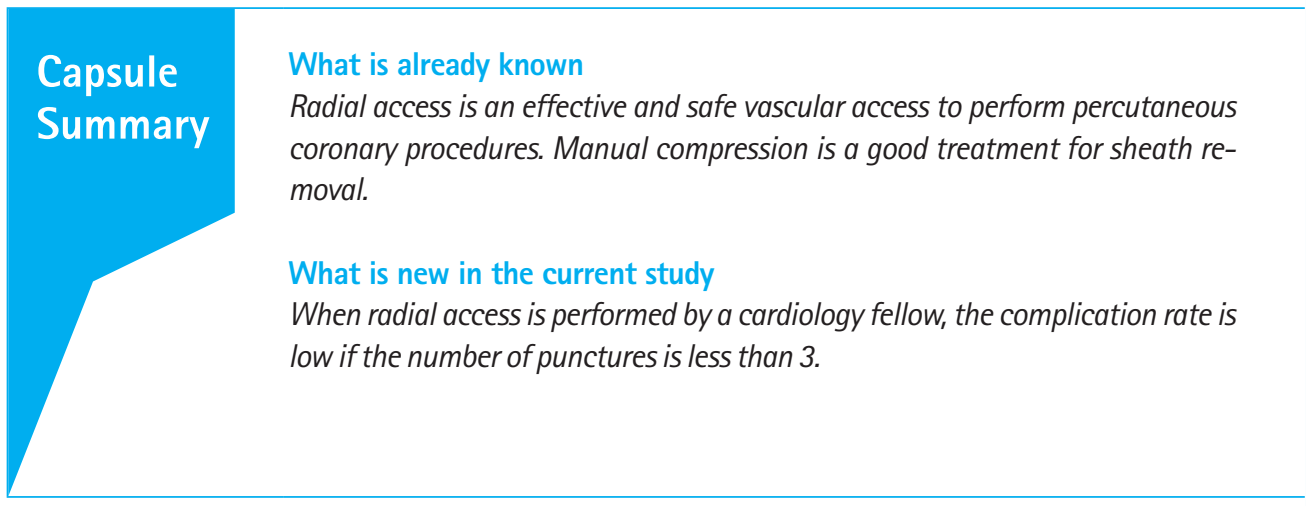

elSSN: 2383-4625

Received: 6 February 2017

Revised: 11 April 2017

Accepted: 13 July 2017

Correspondence to: Marco Angelillis Vascular and Cardio-Thoracic Department, University of Pisa, Via Paradisa 2, Pisa 56100, Italy E-mail: angelillismarco@gmail.com

*These authors contributed equally to the study.

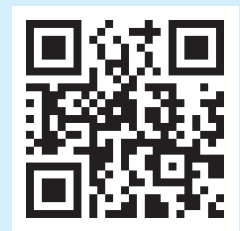

How to cite this article:

Susanu S, Angelillis M, Giannini C, Binella R, Matteoni A, Bellucci R, Balestri S, Ferrara N, Falchi F, Micheletti G, Petronio AS. Radial access for percutaneous coronary procedure: relationship between operator expertise and complications. Clin Exp Emerg Med 2018;5(2):95-99.

This is an Open Access article distributed under the terms of the Creative Commons Attribution Non-Commercial License (http:// creativecommons.org/licenses/by-nc/4.0/). 


\section{INTRODUCTION}

Currently, percutaneous coronary intervention ( $\mathrm{PCl}$ ) is the most used procedure to treat coronary artery disease, both for acute coronary syndrome ${ }^{1,2}$ and for stable coronary artery disease. ${ }^{3}$ The development of a new device and technique for $\mathrm{PCl}$ had led to a decrease in the incidence of adverse event associated with the procedure. A new access-site technique, such as radial access, facilitated a decrease in the incidence of vascular complications related to femoral access, which are associated with poor clinical outcomes at short- and long-term follow-up. ${ }^{4}$ The recent multicenter randomized trial MATRIX ${ }^{5}$ (Minimizing Adverse Hemorrhagic Events by Transradial Access Site and Systemic Implementation of Angiox) showed that radial access reduced the net adverse clinical events through decreases in the incidence of major bleeding and all-cause mortality. An emergent problem in the modern catheterization laboratory is the training of new operators. Experienced operators started to work with femoral access, which can be more difficult to manage especially for bleeding complications. Nowadays, cardiology fellows start their training directly with radial access, which is the first choice of access site in almost all catheterization laboratories, without the background of femoral access. In 2015, 71\% of the interventional procedures performed in Italian catheterization laboratories were carried out through a radial access. Radial access is a more breakable access, even if the related complications are less serious. Operators with no experience in vascular access often can determinate an increase in the incidence of vascular complications or access failure. ${ }^{6}$ These complications often derive from the management of the access after the procedure by introducer removal and access compression, which could be performed manually or with a device. ${ }^{7-9}$

The Catheterization Laboratory, University of Pisa is both a highvolume center and an academic catheterization laboratory, located in a post-graduate school of cardiovascular medicine. We performed 1,446 coronary procedures in 2015, of which 77\% were performed through a radial access. Every 3 months, new cardiology fellows, often with no previous experience in percutaneous vascular access, begin their training. Furthermore, in our center, introducer sheath removal is performed by a skilled nurse at the end of the procedure.

We conducted this study to investigate how the learning curve of cardiology fellows and the management of the access without a specific device affect the incidence of radial access complications.

\section{METHODS}

In the present study, we included all consecutive patients who underwent coronary angiography and/or $\mathrm{PCl}$ with radial access in our center. At the beginning of the procedure, an experienced operator evaluated the radial artery of the wrist with a semiqualitative scale ranging from 0 to 4 , with 0 defined as the absence of a palpable artery, 1 defined as a weakly palpable artery with an unevaluable course, 2 defined as a highly palpable wrist artery with an unevaluable course, 3 defined as a weakly palpable wrist artery with an evaluable course, and 4 defined as a highly palpable wrist artery with an evaluable course. Four cardiology fellows were involved in the study, all without prior experience with radial access. Signed informed consent was obtained from all patients before the procedure. Ethical committee approval was waived for this study because all invasive procedures represent the current best clinical practice in our department.

The time to obtain access was calculated from the puncture for anesthesia to sheath insertion. Furthermore, we recorded all attempts to access the vessel irrespective of bleeding and classified the attempts according to three possible outcomes as follows: puncture of the access site without bleeding caused by the needle, puncture of the access site with bleeding caused by the needle but with inability to advance the wire, and puncture with bleeding caused by the needle and success to cross the wire. Then, the procedure was performed according to the clinical indication. The crossover access to the contralateral or femoral access was not considered as a failure if it depended on the anatomical complexity of the subclavian axis. The introducer sheath used was the Avanti+ transradial radial sheath (Cordis, Bridgewater, NJ, USA). Data about the procedure, such as the number of diagnostic or guide catheters, the total amount of heparin used during the procedure, and the total procedure time, were collected for all the patients. At the end of the procedure, a nurse removed the introducer and bandaged the radial access with a tight bandage. Then, the patient remained 30 seconds in the observation room, and a nurse provided a first loosening of the bandage to check for bleeding. If the hemostasis was good, a new less-tight bandage was prepared and the patient was returned to the ward, with a complete removal of the bandage after 4 hours. Otherwise, if the access was bleeding, a new tight bandage was applied, always with a complete removal after 4 hours.

The primary end point was a composite of pulseless radial artery of the wrist and hematoma evaluated after 24 hours (late complications). We performed statistical analyses to identify the minimum number of punctures needed to reduce adverse events. The secondary end point of efficacy was defined as the presence of bleeding or hematoma after 30 minutes (early complications).

Continuous variables were presented as mean \pm standard deviation or as median and interquartile range, and compared using 
the Student t-test or Mann-Whitney test as appropriate. Categorical variables were presented as frequency and percentage, and compared using the Fisher exact test. A 2-tailed P-value of $<0.05$ was considered significant. Statistical analyses were performed using IBM SPSS Statistics ver. 23.0 (IBM Corp., Armonk, NY, USA).

\section{RESULTS}

The patients' baseline characteristics are summarized in Table 1 . From March 2016 to June 2016, 150 consecutive patients who underwent coronary angiography and/or PCl through radial access were included in the present study. During this period, four cardiology fellows rotated through the catheterization laboratory. Thus, we can assume a mean of 37 cases for each fellow. All the fellows had no prior experience with percutaneous vascular access. The mean age of the patients was $70.1 \pm 12.7$ years. Of the patients, $67.3 \%(n=101)$ were male. In $56.7 \%(n=85)$ of the cases, the patient presented with an acute coronary syndrome, which was an ST-segment elevation myocardial infarction in 43.5\% of the cases. The procedural characteristics are summarized in Table 2. After radial access evaluation, we classified the radial ar-

Table 1. Patients' baseline characteristics

\begin{tabular}{lc}
\hline Baseline characteristic & Value \\
\hline Age (yr) & $70.1 \pm 12.7$ \\
Sex, male & $101 / 150(67.3)$ \\
BMI $\left(\mathrm{kg} / \mathrm{m}^{2}\right)$ & $27.5 \pm 4.5$ \\
SAP at start procedure & $126.4 \pm 15.9$ \\
DAP at start procedure & $73.3 \pm 10.3$ \\
PCl performed & $62 / 150(41.3)$ \\
Hypertension & $124 / 150(82.6)$ \\
Diabetes & $48 / 150(32)$ \\
Smoker & $53 / 150(35.3)$ \\
Previous myocardial infarction & $6 / 150(4)$ \\
Peripheral artery disease & $21 / 150(14)$ \\
Previous PCl with radial access & $8 / 150(5.3)$ \\
ACS patients & $85 / 150(56.7)$ \\
STEMI & $37 / 85(43.5)$ \\
NSTEMI/UA & $48 / 85(56.5)$ \\
SCAD & $65 / 150(43.3)$ \\
No. of cardiology fellows involved in the study) & 4 \\
Mean number of patients for each fellow & 37.5
\end{tabular}

Values are presented as number (\%) or mean \pm standard deviation.

$\mathrm{BMI}$, body mass index; SAP, systolic arterial pressure; DAP, diastolic arterial pressure; $\mathrm{PCl}$, percutaneous coronary intervention; $\mathrm{ACS}$, acute coronary syndrome; STEMI, ST-segment elevation myocardial infarction; NSTEMI, non-ST-segment elevation myocardial infarction; UA, unstable angina; SCAD, stable coronary artery disease.

a)All cardiology fellows were at their first experience with vascular access. tery of the wrist with values of 1,2, 3, and 4 in 3.3\%, 19.4\%, 24\%, and $53.3 \%$ of the patients, respectively. A successful access was achieved in $95.3 \%$ of the cases. The mean access time was 4.5 minutes. The diagnostic procedure was performed in 88 patients (58.6\%), whereas 17 patients underwent PCl directly, usually for a staged revascularization. The mean number of diagnostic catheters for systematic use in the dual catheter technique for diagnostic angiography was 2.23. Regarding the needle puncture, four or more attempts were needed in only $10.7 \%$ of the patients. The access was obtained with a single puncture in $60 \%$ of the patients, even though it was performed by training physicians.

Early complications (within half an hour) developed in 16 patients (10.7\%), including bleeding related to the access site $(n=7)$ and hematoma $(n=9)$. A new bandaging was needed in 7 patients $(4.6 \%)$, while in the remaining patients, the previous bandaging was loosened in accordance with the protocol. Late complications (after 24 hours) occurred in 30 patients (20\%) and were all cases of hematoma. In 5 patients (3.3\%), the wrist artery was not palpable. The primary and secondary efficacy end points occurred in $33(22 \%)$ and 7 patients $(4.7 \%)$, respectively. Furthermore, we found that having fewer than four punctures to obtain vascular access was significantly associated with a lower incidence of pri-

Table 2. Procedural characteristics

\begin{tabular}{lc}
\hline Procedural characteristic & Value \\
\hline Radial evaluation & \\
1 & $5 / 150(3.3)$ \\
2 & $29 / 150(19.3)$ \\
3 & $36 / 150(24)$ \\
4 & $80 / 150(53.3)$ \\
Median access time (min) & $4.5(2-5)$ \\
No. of punctures & $1.82 \pm 1.3$ \\
1 & $90(60)$ \\
2 & $28(18.7)$ \\
3 & $16(10.7)$ \\
4 & $6(4)$ \\
5 & $6(4)$ \\
6 & $3(2)$ \\
7 & $1(0.7)$ \\
No. of introducers used & $1.02 \pm 0.3$ \\
No. of diagnostic catheters used & $2.2 \pm 1.2$ \\
Angiography without PCl & $88(58.6)$ \\
PCl & $62(41.4)$ \\
No. of guide catheters used & $1.24 \pm 0.4$ \\
Mean heparin dose (UI) & $6,160 \pm 2.481 .7$ \\
Procedure time (min) & $47.4 \pm 32.6$ \\
No. of successful access & $143 / 150(95.3)$ \\
\hline & \\
\hline &
\end{tabular}

Values are presented as number (\%), median (interquartile range), or mean \pm standard deviation.

$\mathrm{PCl}$, percutaneous coronary intervention. 
Table 3. OR of primary end point according to the number of punctures

\begin{tabular}{|c|c|c|c|c|c|}
\hline \multicolumn{3}{|c|}{ No. of puncture } & \multirow{2}{*}{$\begin{array}{c}\begin{array}{c}\text { Primary end } \\
\left.\text { point }{ }^{2}\right) \\
(n=33)\end{array} \\
9(56.2)\end{array}$} & \multirow{2}{*}{$\begin{array}{c}\text { OR } \\
(95 \% \mathrm{Cl}) \\
3.14(1.78-5.52)\end{array}$} & \multirow{2}{*}{$\begin{array}{r}\text { P-value } \\
0.002\end{array}$} \\
\hline \multirow[t]{4}{*}{ Puncture ${ }^{\text {b) }}$} & $\geq 4(n=16)$ & & & & \\
\hline & $<4(n=132$ & & 24 (17.9) & & \\
\hline & $\geq 3(n=32)$ & & $10(31.2)$ & $1.60(0.85-3.01)$ & 0.158 \\
\hline & $<3(n=118$ & & 23 (19.5) & & \\
\hline \multirow{4}{*}{\multicolumn{2}{|c|}{ Puncture with blood ${ }^{c}$}} & $\geq 4(n=4)$ & $3(75)$ & $3.65(1.90-6.98)$ & 0.034 \\
\hline & & $<4(n=146)$ & $1(0.7)$ & & \\
\hline & & $\geq 3(n=6)$ & $4(66.7)$ & $3.31(1.72-6.35)$ & 0.021 \\
\hline & & $<3(n=144)$ & $29(20.1)$ & & \\
\hline
\end{tabular}

Values are presented as number (\%).

$\mathrm{OR}$, odds ratio; $\mathrm{Cl}$, confidence interval.

a) The primary end point is a composite of pulseless radial wrist and hematoma evaluated after 24 hours. ${ }^{b}$ Refers to the total number of punctures regardless of the presence of blood. 'The total number of punctures with blood but no possibility to advance wire.

mary end point when compared with four or more punctures (17.9\% vs. 56.2\%; odds ratio, $3.14 ; 95 \%$ confidence interval, 1.78 to $5.52 ; P=0.002$ ) (Table 3). No correlation between the primary and secondary end points was highlighted in our analyses. Otherwise, considering only puncture with successful blood flow but no possibility of advancing the wire, we found that fewer than three punctures were associated with a lower incidence of complications as compared with three or more punctures $(20.1 \%$ vs. $66.7 \%$; odds ratio, $3.31 ; 95 \%$ confidence interval, 1.72 to 6.35 ; $\mathrm{P}=0.021$.

\section{DISCUSSION}

Cardiology fellowship apprentices significantly support patient care in academic medical centers. This includes performance of invasive procedures in the cardiac catheterization laboratory. Major studies that compared radial and femoral access techniques have focused on data obtained from skilled and experienced operators; similar data with cardiology fellows as primary operators are limited.

The major findings from our study include the following: (1) radial access is a quick vascular access and feasible also by cardiology fellows; (2) three or more puncture attempts (when considering successful blood-taking puncture but without wire advancement) are associated with an increase in the rate of complications; and (3) management of access bandage with a manual system provides good results in terms of hemostasis and complications.

Radial access for $\mathrm{PCl}$ is the most investigated technical solution to reduce bleeding and vascular complications, especially for populations with a high bleeding risk, such as women. The previous studies, including a randomized clinical trial, showed how ra- dial access reduced bleeding and influenced long-term outcome. ${ }^{10-12}$ An emergent issue is that cardiology fellows start their training directly with radial access without a knowledge background on femoral access, considering that radial access is prevalently used in catheterization laboratories.

In our study, the success rate of radial access was more than $95 \%$, even though it was performed by training operators. The median time to obtain access was approximately 4.5 minutes, lower than that reported in a previous study that evaluated cardiology fellows. ${ }^{13}$ Other interesting data that emerged from this analysis are that in more than 60\% of patients, radial access was obtained at first attempt and, if the operator was in training, underline the feasibility of this vascular access.

From our observations, three or more puncture attempts seemed to not increase the complication rate, so training physicians could be allowed to try to obtain access until these limits. However, when we consider only punctures touching the artery, this limit decreases to two attempts. This finding underlines that only puncture touching the artery can lead to an increase in complication rate, whereas puncture of subcutaneous space is not associated with arterial complications. The incidence of radial occlusion evaluated by palpation in our study was $3.3 \%$ at 24 hours, lower than the $7.7 \%$ reported in a recent meta-analysis. ${ }^{14}$

Management of the radial access site after the procedure is controversial. Different studies investigated whether the use of a bandaging device is more effective than use of a bandage alone, but no clear evidence has emerged. ${ }^{8,15,16}$ Previous studies reported that short-time compression is equally effective in terms of radial occlusion and complication rates..$^{7.9}$ In our center, bandaging with an elastic compression band represents the standard procedure to remove the introducer. In addition, we performed an early evaluation of the access site after 30 minutes to evaluate whether hemostasis was obtained. With these protocols, we obtained a $3.3 \%$ incidence rate of severe complications, represented by a pulseless wrist at 24 hours. The incidence of other complications such as the hematoma was less substantial, and none led to a disability or prolonged hospitalization.

This study is constrained by the limitations inherent to a nonrandomized study. Our sample is from a single center and limited to a relatively short time frame. In addition, variables such as patient satisfaction and comfort were not included owing to the study design. This finding could have been a result of the suitable selection of patients by attending physicians for fellowship trainees to gain vascular access. Despite these limitations, our study has potential implications in guiding fellowship training curriculum by demonstrating that transradial access poses a steeper learning curve for cardiology fellows. Another limitation of the 
study is the absence of a long-term follow-up of the access site (i.e., at 1 month) and of an objective evaluation to determine the radial occlusion.

Radial access represents an ideal vascular access for $\mathrm{PCl}$ and for unskilled physicians. In hospitals with training programs for $\mathrm{PCl}$, no more than three (or two with successful drawing of blood) punctures should be attempted to avoid an increase in vascular complications. A manual bandage with early examination for bleeding can be used to manage the access site after the intervention.

\section{CONFLICT OF INTEREST}

No potential conflict of interest relevant to this article was reported.

\section{REFERENCES}

1. Biondi-Zoccai GG, Abbate A, Agostoni P, et al. Long-term benefits of an early invasive management in acute coronary syndromes depend on intracoronary stenting and aggressive antiplatelet treatment: a metaregression. Am Heart J 2005;149: 504-11.

2. Barringhaus KG, Akhter M, Rade JJ, Smith C, Fisher DZ. Operator and institutional experience reduces room-to-balloon times for transradial primary percutaneous coronary intervention. J Invasive Cardiol 2014;26:80-6.

3. Piccolo R, Giustino G, Mehran R, Windecker S. Stable coronary artery disease: revascularisation and invasive strategies. Lancet 2015;386:702-13.

4. Chhatriwalla AK, Amin AP, Kennedy KF, et al. Association between bleeding events and in-hospital mortality after percutaneous coronary intervention. JAMA 2013;309:1022-9.

5. Valgimigli $M$, Gagnor $A$, Calabro $P$, et al. Radial versus femoral access in patients with acute coronary syndromes undergoing invasive management: a randomized multicentre trial. Lancet 2015;385:2465-76.

6. Ball WT, Sharieff W, Jolly SS, et al. Characterization of operator learning curve for transradial coronary interventions. Circ Cardiovasc Interv 2011;4:336-41.
7. Pancholy SB, Patel TM. Effect of duration of hemostatic compression on radial artery occlusion after transradial access. Catheter Cardiovasc Interv 2012;79:78-81.

8. Pancholy SB. Impact of two different hemostatic devices on radial artery outcomes after transradial catheterization. J Invasive Cardiol 2009;21:101-4.

9. Politi L, Aprile A, Paganelli $C$, et al. Randomized clinical trial on short-time compression with Kaolin-filled pad: a new strategy to avoid early bleeding and subacute radial artery occlusion after percutaneous coronary intervention. J Interv Cardiol 2011;24:65-72.

10. Mack MJ, Leon MB, Smith CR, et al. 5-year outcomes of transcatheter aortic valve replacement or surgical aortic valve replacement for high surgical risk patients with aortic stenosis (PARTNER 1): a randomised controlled trial. Lancet 2015;385: 2477-84.

11. Ferrante $G$, Rao SV, Juni $P$, et al. Radial versus femoral access for coronary interventions across the entire spectrum of patients with coronary artery disease: a meta-analysis of randomized trials. JACC Cardiovasc Interv 2016;9:1419-34.

12. Huang $F Y$, Huang BT, Wang PJ, et al. Gender disparity in the safety and efficacy of radial and femoral access for coronary intervention: a systematic review and meta-analysis. Angiology 2016;67:810-9.

13. Singh G, Campos E, Kavinsky CJ, Snell JR, Schaer GL, Jolly N. Comparative analysis of radial versus femoral diagnostic cardiac catheterization procedures in a cardiology training program. J Invasive Cardiol 2016;28:254-7.

14. Rashid M, Kwok CS, Pancholy S, et al. Radial artery occlusion after transradial interventions: a systematic review and metaanalysis. J Am Heart Assoc 2016 Jan 25. https://doi.org/10.1161/ JAHA. 115.002686.

15. Yun K, Jeon W, Kang B, Kim G. Effectiveness of a compressive device in controlling hemorrhage following radial artery catheterization. Clin Exp Emerg Med 2015;2:104-9.

16. Sanborn TA, Gibbs HH, Brinker JA, Knopf WD, Kosinksi E, Roubin GS. A multicenter randomized trial comparing a percutaneous collagen hemostasis device with conventional manual compression after diagnostic angiography and angioplasty. J Invasive Cardiol 1999;11 Suppl B:6B-13B. 